\title{
Noise-Modulated Effects of Anisotropy and Position- Dependent Effective Mass on the Oscillator Strength of Impurity Doped Quantum Dots
}

\author{
Sucharita Sarkar ${ }^{1}$, Arghya Pratim Ghosh², Arkajit Mandal ${ }^{3}$ and Manas Ghosh ${ }^{2}$ \\ ${ }^{1}$ Department of Chemistry and Biochemistry,University of Delaware, Newark, Delaware 19716, USA \\ ${ }^{2}$ Department of Chemistry, Physical Chemistry Section, Visva Bharati University, Santiniketan, Birbhum \\ 731235, West Bengal, India. \\ ${ }^{3}$ Department of Chemistry, University of Rochester, New York 14627, USA \\ Email: pcmg77@rediffmail.com
}

\begin{abstract}
We study the modulation of oscillator strength (OS) of impurity doped quantum dot (QD) under the influence of geometrical anisotropy and position-dependent effective mass (PDEM) in presence and absence of noise. The OS profiles are monitored as a function of anisotropy and dopant location considering PDEM and fixed effective mass (FEM). Noise considered here is Gaussian white noise which has been administered to the system additively and multiplicatively. Always a comparison has been attempted between FEM and PDEM to understand the role of the latter on OS profiles. Application of noise has been found to affect the OS profiles only over some particular domains of anisotropy and dopant location. And use of PDEM promotes greater contribution from noise than FEM in fabricating the OS profiles. The observations reveal sensitive interplay between noise and anisotropy/PDEM to tailor the features of OS profiles which bear substantial technological importance in the study of nonlinear optical properties of doped QD systems.
\end{abstract}

Keywords: Quantum dot, impurity, Gaussian white noise, oscillator strength, geometrical anisotropy, position-dependent effective mass

\section{Introduction}

Low-dimensional semiconductor systems (LDSS) such as quantum wells (QWLs), quantum wires (QWRs) and quantum dots (QDs) are widely appreciated for their noticeably large nonlinear optical (NLO) properties. The enhanced quantum confinement effect (in comparison with the bulk materials) existing in LDSS favors such magnified nonlinear effects [1].Such strong confinement in LDSS causes small energy interval between the subband levels and large value of electric dipole matrix elements. These two factors promote achievement of resonance conditions. Such enhanced NLO properties of LDSS turn out to be immensely significant in view of probing the electronic structure of mesoscopic media [2], application of electronic and optoelectronic devices in the infra-red region of the electromagnetic spectrum [3, 4], exploring the area of integrated optics and optical communications $[5,6]$, fabricating many optoelectronic devices such as far-IR laser amplifiers, photo-detectors, and high-speed electro-optical modulators [7-9] and most significantly, understanding and realization of fundamental physics.

Oscillator strength $(O S)$ is an extremely significant dimensionless quantity absolutely pertinent to the investigation of optical properties which are connected to the electronic dipole allowed transitions. It also delivers additional information regarding the fine structure and selection rules of optical absorption. In LDSS large value of OS is responsible for high dipole moment expectation values and consequent exhibition of large NLO response. These important features have led to some prominent theoretical researches on OS by Yilmaz and Şafak [10], Özmen et al. [11], Çakir et al. [12], Kumar et al. [13], Tiutiunnyk et al. [14], Xie [15], Sadeghi [16], and He and Xie [17], to mention a few. 
Ingression of impurity (dopant) into LDSS initiates delicate interplay between the dopant potential with the confinement potential of LDSS and eventually modifies the energy level distribution. Such modification severely affects the electronic and optical properties of LDSS. Thus, a well-controlled inclusion of dopant favors achievement of desirable optical transitions. Such desirable optical transition has become an essential part of fabricating optoelectronic devices with tunable emission or transmission properties and ultra narrow spectral line widths. This has largely opened up new vista of technological applications of LDSS. Moreover, the proximity of optical transition energy and the confinement strength (or the quantum size) can effectively design the resonance frequency. In what follows, optical properties of doped LDSS have envisaged rigorous research activities [18-49].

Of late, we have come across a few important studies concerned with the influence of geometrical anisotropy on the optical properties of LDSS. Among them the important contributions were made by Niculescu et al. [39], Xie and his coworkers [50-52] and Safarpour et al. [53, 54]. In reality, in most cases LDSS are not at all isotropic which justifies the need of realizing how anisotropy governs their optical properties. In practice anisotropic QDs can be manufactured by chemically controlling the nanostructure aspect ratio [50]. Thus, study of anisotropic systems has produced substantial interest in view of obtaining novel as well as useful devices.

In recent times we also envisage a considerable number of investigations which involve positiondependent effective mass (PDEM) of LDSS. PDEM gives rise to perceptible change in the binding energy of the doped system and thus alters the optical properties. Such change in the optical properties has induced lots of studies on LDSS with spatially varying effective mass. With reference to above the works of Rajashabala and Navaneethakrishnan [55-57], Peter and Navaneethakrishnan [58], Khordad [59, 60], Qi et. al. [61], Peter [62], Li et. al. [63], and Naimi et. al. [64] deserve attention.

Presence of noise invariably affects the NLO properties of mesoscopic devices. Motivated by this fact recently we have made thorough investigations on how Gaussian white noise affects the oscillator strength of doped QD [65]. We have also explored the role played by geometrical anisotropy [66] and PDEM [67, 68] on various NLO properties of doped QD in presence of noise. However, despite a thorough literature survey we have not found any study that deals with influence of noise on OS under the purview of anisotropy and/or PDEM. Realizing the fact that OS forms the backbone of emergence of many NLO properties, in the present work we explore the influence of geometrical anisotropy and PDEM on OS of doped QD in presence of Gaussian white noise. The OS profiles are monitored for different extents of geometrical anisotropy (to understand the anisotropy effect) and simultaneously with fixed effective mass (FEM) and dopant position-dependent effective mass (PDEM) (to understand the role of PDEM). Moreover, the influence of pathway of application of noise (additive/multiplicative) has also been explored for a comprehensive analysis.

\section{$2 \quad$ Method}

The impurity doped QD Hamiltonian, subject to external static electric field (F) applied along $x$ and $y$ directions and noise (additive/multiplicative) can be written as

$$
H_{0}=H_{0}^{\prime}+V_{\text {imp }}+|e| F(x+y)+V_{\text {noise }}
$$

Under effective mass approximation, $H_{0}^{\prime}$ represents the impurity-free 2-d quantum dot containing single carrier electron under lateral parabolic confinement in the $x-y$ plane and in presence of a perpendicular magnetic field. $\mathrm{V}_{\mathrm{imp}}$ is the impurity (dopant) potential and $V_{\text {noise }}$ stands for white noise applied to the system. $H_{0}^{\prime}$ is therefore given by $[69,70]$.

$$
H_{0}^{\prime}=\frac{1}{2 m^{*}}\left[-i \hbar \nabla+\frac{e}{c} A\right]^{2}+\frac{1}{2} m^{*} \omega_{0}^{2}\left(x^{2}+y^{2}\right)
$$

$m^{*}$ represents the effective mass of the electron inside the QD material. $e$ and $c$ are charge of electron and velocity of light, respectively. $V(x, y)=\frac{1}{2} m^{*} \omega_{0}^{2}\left(x^{2}+y^{2}\right)$ is the confinement potential with $\omega_{0}$ as the harmonic confinement frequency. Using Landau gauge $\left[A=\left(B_{y}, 0,0\right)\right.$, where $A$ is the vector potential and $\mathrm{B}$ is the magnetic field strength], $H_{0}^{\prime}$ reads 


$$
H_{0}^{\prime}=-\frac{\hbar^{2}}{2 m^{*}}\left(\frac{\partial^{2}}{\partial x^{2}}+\frac{\partial^{2}}{\partial y^{2}}\right)+\frac{1}{2} m^{*} \omega_{0}^{2} x^{2}+\frac{1}{2} m^{*}\left(\omega_{0}^{2}+\omega_{c}^{2}\right) y^{2}-i \hbar \omega_{c} y \frac{\partial}{\partial x}
$$

$\omega_{c}=\frac{e B}{m^{*} c}$ being the cyclotron frequency. $\Omega=\sqrt{\omega_{0}^{2}+\omega_{c}^{2}}$ can be viewed as the effective confinement frequency in the y-direction. Following the notable works of Xie the ratio $\eta=\frac{\Omega}{\omega_{0}}$ could be defined as the anisotropy parameter [50-52].

$\mathrm{V}_{\mathrm{imp}}$ is represented by a Gaussian function [65] i.e. $V_{i m p}=V_{0} e^{-\gamma\left[\left(x-x_{0}\right)^{2}+\left(y-y_{0}\right)^{2}\right]}\left(x_{0}, y_{0}\right), V_{0}$ and $\gamma^{-\frac{1}{2}}$ are the site of dopant incorporation, strength of the dopant potential, and the spatial spread of impurity potential, respectively. $\gamma$ can be written as $\gamma=k \varepsilon$, where $k$ is a constant and $\varepsilon$ is the dielectric constant of the medium.

The dopant location-dependent effective mass $m^{*}\left(r_{0}\right)$ where $r_{0}=\sqrt{x_{0}^{2}+y_{0}^{2}}$ is given by $[55,58]$

$$
\frac{1}{m^{*}\left(r_{0}\right)}=\frac{1}{m^{*}}+\left(1-\frac{1}{m^{*}}\right) \exp \left(-\beta r_{0}\right)
$$

where $\beta$ is a constant chosen to be 0.01 a.u. The choice of above form of PDEM indicates that the dopant is strongly bound to the dot confinement center as $r_{0} \rightarrow 0$ i.e. for on-center dopants whereas $m^{*}\left(r_{0}\right)$ becomes highly significant as $r_{0} \rightarrow \infty$ i.e. for far off center dopants.

$V_{\text {noise }}$ stands for white noise $[f(x, y)]$ which follows a Gaussian distribution (generated by BoxMuller algorithm), has a strength $\zeta$ and is characterized by zero-average and spatial $\delta$-correlation conditions [65-67]. Such white noise can be introduced to the system via two different modes (pathways) i.e. additive and multiplicative [65-67]. These two different modes can be discriminated on the basis of extent of system-noise interaction.

The time-independent Schrödinger equation has been solved by generating the sparse Hamiltonian matrix $H_{0}$. The relevant matrix elements involve the function $\psi(x, y)$ which is a superposition of the products of harmonic oscillator eigenfunctions. In this context a sufficient number of basis functions have been included after performing the convergence test. $H_{0}$ is diagonalized afterwards in the direct product basis of harmonic oscillator eigenfunctions to obtain the energy levels and wave functions.

OS is given by $[10-17]$

$$
P_{i j}=\frac{2 m^{*}}{\hbar^{2}} \Delta E_{i j}\left|M_{i j}\right|^{2}
$$

where $\Delta E_{i j}=E_{j}-E_{i}$ is the energy difference between states $|i\rangle$ and $|j\rangle$ and $M_{i j}=\left\langle\psi_{i}|\hat{x}+\hat{y}| \psi_{j}\right\rangle$ is the electric dipole transition moment. The matrix elements are usually small whereas $\Delta E_{i j}$ is very high in small QDs.

\section{Results and Discussion}

The calculations are performed using the following parameters: $\varepsilon=12.4, \mathrm{~m}^{*}=0.067 \mathrm{~m}_{0}$, where $\mathrm{m}_{0}$ is the free electron mass, $\hbar \omega_{0}=2.72 \mathrm{meV}, \mathrm{F}=100 \mathrm{KV} / \mathrm{cm}, \mathrm{B}=1.0 \mathrm{~T}, \zeta=1.0 \times 10^{-13}, \mathrm{~V}_{0}=272.0 \mathrm{meV}$. The parameters are suitable for GaAs QDs.

Role of anisotropy $(\eta)$ : Fig. 1 shows the variations of OS with anisotropy parameter $\eta$ in absence of noise [fig. 1(i)] and in presence of additive [fig. 1(ii)] and multiplicative [fig. 1(iii)] noise, respectively. Up to $\eta \sim 25$ OS remains nearly unobservable. However, as soon as $\eta \geq 25$, OS exhibits pronounced enhancement with increase in $\eta$ and culminates into saturation beyond $\eta \sim 175$. The profiles have been found to be nearly identical both in presence and absence of noise throughout the entire range of variation of $\eta$ with minor fluctuations. However, only in the neighborhood of a typical 
anisotropy regime i.e. $\eta \sim 28$ a somewhat observable noise induced departure of the OS profiles has been found from that of noise-free situation [66]. The mode of application of noise (additive/multiplicative) comes out to be quite inactive in modulating the OS profile.

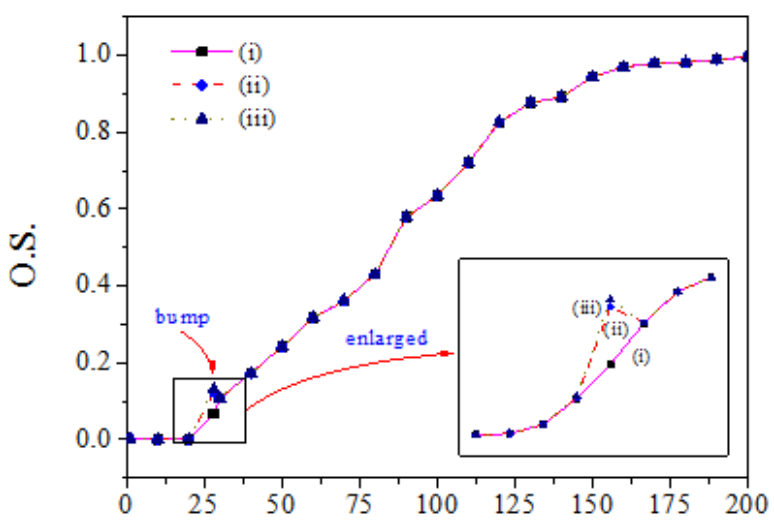

Figure. Plots of OS vs $\eta$ : (i) under noise-free condition, (ii) in presence of additive noise and (iii) in presence of multiplicative noise.

The observations indicate that a fruitful overlap between concerned eigenstates can only be realized if and only if $\eta \geq 25$ both in presence and absence of noise. As soon as anisotropy exceeds the threshold value of $\eta \sim 25$ the said overlap comes into play and begins to increase profoundly with increase in $\eta$. And within very large anisotropy domain of $\eta \geq 175$ the overlap settles to some steady value. Moreover, application of noise happens to moderately enhance the said overlap only through a small anisotropy window (in the vicinity of $\eta \geq 25$ ) and fails to make any impact onthe entire remaining anisotropy domain.

Role of PDEM: Fig. 2 evinces the variation of OS with dopant location $\left(r_{0}\right)$ using PDEM $\left[m^{*}\left(r_{0}\right)\right]$ in absence of noise [fig. 2(i)] and in presence of additive [fig. 2(ii)] and multiplicative [fig. 2(iii)] noise, respectively. In absence of noise the OS profile exhibits successive maximization and minimization at $r_{0}$ $=0.1 \mathrm{~nm}$ and $r_{0}=0.3 \mathrm{~nm}$, respectively. After minimization OS increases with $r_{0}$ and shows some sort of steady value beyond $r_{0}=0.6 \mathrm{~nm}$.

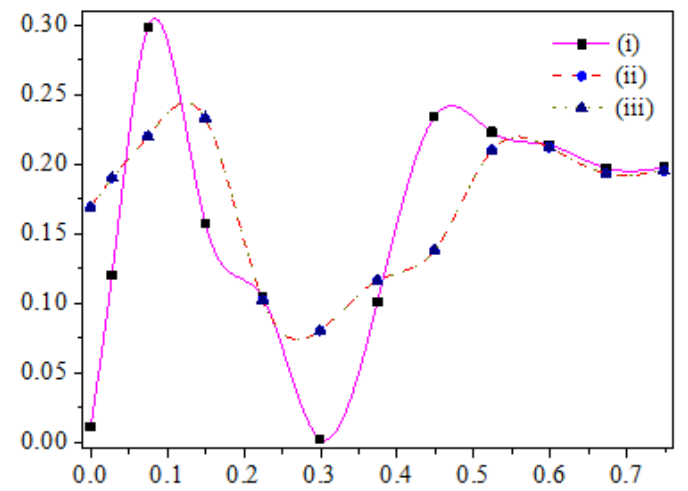

Figure 2. Plots of OS vs $\mathrm{r}_{0}$ using PDEM: (i) under noise-free condition, (ii) in presence of additive noise and (iii) in presence of multiplicative noise.

The profile suggests that, in absence of noise, PDEM of dopant causes maximum and minimum overlap between the relevant eigenfunctions at some typical dopant locations and the extent of overlap 
attains some stability for the off-center dopants. Application of noise does not much alter the overall OS profile from that of noise-free condition qualitatively. However, it is the magnitude of OS which is affected most. The plot reveals noise-induced suppression and amplification of OS (in comparison with noise-free case) nearly at the same dopant locations where maximization and minimization have been found previously. And beyond $\mathrm{r}_{0}=0.6 \mathrm{~nm}$ the noise effect nearly subsides and the OS value approaches the noise-free one. It can therefore be inferred that presence of noise simply modulates the size of overlap between the pertinent wave functions from that of noise-free condition. Noise, however, does not grossly affect the pattern of said overlap as a function of dopant location in case of PDEM. The mode of application of noise, as before, does not exhibit any noticeable contribution.

Role of FEM: Fig. 3 displays the variation of OS with dopant location $\left(r_{0}\right)$ using FEM $\left(m^{*}=\right.$ $0.067 m_{0}$ ) in absence of noise [fig. 3(i)] and in presence of additive [fig. 3(ii)] and multiplicative [fig. 3(iii)] noise, respectively. Both in presence and absence of noise the OS profiles exhibit maximization around a dopant location of $\mathrm{r}_{0}=0.3 \mathrm{~nm}$ indicating maximum overlap between the eigenstates concerned. It needs to be noted that the difference between OS profiles in absence and presence of noise decreases further using FEM than using PDEM. In the present case of FEM, only for on-center $\left(\mathrm{r}_{0}=0.0 \mathrm{~nm}\right)$ and very near off-center $\left(\mathrm{r}_{0} \leqslant 0.1 \mathrm{~nm}\right)$ dopants, presence of noise causes prominent amplification of OS over that of noise-free condition. At all other dopant locations noise remains insignificant.

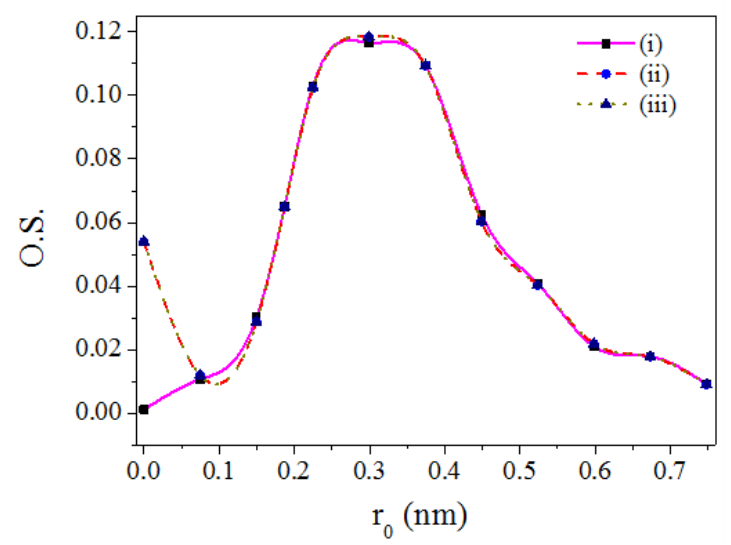

Figure 3. Plots of OS vs $\mathrm{r}_{0}$ using FEM: (i) under noise-free condition, (ii) in presence of additive noise and (iii) in presence of multiplicative noise.

\section{Conclusion}

The modulation of oscillator strength (OS) of impurity doped QD has been investigated under the influence of geometrical anisotropy and position-dependent effective mass (PDEM) in presence and absence of noise. The findings can be summarized as follows:

1. OS becomes noticeable only if the anisotropy parameter $\eta$ exceeds a threshold value of $\sim 25$ both in presence and absence of noise. Effect of noise on OS profile can be manifested only around a narrow anisotropy domain of $\eta \sim 28$ when a moderate enhancement of OS takes place.

2. In case of PDEM, OS undergoes maximization and minimization depending upon the dopant location both in presence and absence of noise. Presence of noise does not qualitatively alter the OS profiles but only affects the extent of maximization and minimization causing suppression and amplification of OS.

3. In case of FEM, OS displays prominent maximization around $\mathrm{r}_{0} \sim 0.3 \mathrm{~nm}$ both in presence and absence of noise. Difference between the OS profiles in absence and presence of noise becomes perceptible only for on-center $\left(\mathrm{r}_{0}=0.0 \mathrm{~nm}\right)$ and very near off-center $\left(\mathrm{r}_{0} \leqslant 0.1 \mathrm{~nm}\right)$ dopants where OS gets amplified in presence of noise. On the whole, it is PDEM thatinvites greater difference between OS profiles in presence and absence of noise than FEM. 
Above features can highlight important aspects of NLO properties of doped QD systems in presence of noise.

Acknowledgements. The authors S. S., A. P. G., A. M. and M. G. thank D. S. T-F. I. S. T (Govt. of India) and U. G. C.- S. A. P (Govt. of India) for their support.

\section{Reference}

1. B. Chen, K. -X. Guo, Z. -L. Liu, R. -Z. Wang, Y. -B. Zheng and B. Li, "Second-order nonlinear optical susceptibilities in asymmetric coupled quantum wells", Journal of Physics: Condensed Matter, vol. 20, no. 25, pp. 255214, 2008.

2. K. -X. Guo, T. P. Das and C. -Y. Chen, "Studies on the electro-optic effects of double-layered quantum wires in magnetic fields", Physica B, vol. 293, no. 1-2, pp. 11-15, 2000.

3. B. Chen, K. -X. Guo, R. -Z. Wang and Z. -H. Zhang, "Optical second harmonic generation in asymmetric double triangular quantum wells", Superlattices and Microstructures, vol. 45, no. 3, pp. 125-133, 2009.

4. İ. Karabulut, "Laser field effect on the nonlinear optical properties of a square quantum well under the applied electric field", Applied Surface Science, vol. 256, no. 24, pp. 7570-7574, 2010.

5. M. E. Mora-Ramos, C. A. Duque, E. Kasapoglu, H. Sari and I. Sökmen, "Linear and nonlinear optical properties in a semiconductor quantum well under intense laser radiation: Effects of applied electromagnetic fields", Journal of Luminescence, vol. 132, no. 4, pp. 901-913, 2012.

6. C. A. Duque, E. Kasapoglu, S. Şakiroğlu, H. Sari and I. Sökmen, "Intense laser effects on nonlinear optical absorption and optical rectification in single quantum wells under applied electric and magnetic field", Applied Surface Science, vol. 257, no. 6, pp. 2313-2319, 2011.

7. S. Şakiroğlu, F. Ungan, U. Yesilgul, M. E. Mora-Ramos, C. A. Duque, E. Kasapoglu, H. Sari and I. Sökmen, "Nonlinear optical rectification and the second and third harmonic generation in Pöschl-Teller quantum well under the intense laser field", Physics Letters A, vol. 376, no. 23, pp. 1875-1880, 2012.

8. F. Ungan, J. C. Mertínez-Orozco, R. L. Restrepo, M. E. Mora-Ramos, E. Kasapoglu and C. A. Duque," Nonlinear optical rectification and second-harmonic generation in a semi-parabolic quantum well under intense laser field: Effects of electric and magnetic fields", Superlattices and Microstructures, vol. 81, no. May, pp. 26-33, 2015.

9. H. Hassanabadi, G. Liu and L. Lu, "Nonlinear optical rectification and the second-harmonic generation in semiparabolic and semi-inverse quantum wells", Solid State Communications, vol. 152, no. 18, pp. 1761-1766, 2012.

10. S. Yilmaz and H. Şafak, "Oscillator Strengths for the intersubband transitions in a $\mathrm{Cds}-\mathrm{SiO}_{2}$ quantum dot with hydrogenic impurity", Physica E, vol. 36, no. 1, pp. 40-44, 2007.

11. A. Özmen, Y. Yakar, B. Çakir and Ü. Atav, "Computation of the oscillator strength and absorption coefficients for the intersubband transitions of the spherical quantum dot", Optics Communications, vol. 282, no. 19, pp. 3999-4004, 2009.

12. B. Çakir, Y. Yakar and A. Özmen, "Calculation of oscillator strength and the effects of electric field on energy states, static and dynamic polarizabilities of the confined hydrogen atom", Optics Communications, vol. 311, no. 15 January, pp. 222-228, 2013.

13. K. M. Kumar, A. J. Peter and C. W. Lee, "Optical properties of a hydrogenic impurity in a confined $Z n_{1-}$ ${ }_{x} C d_{x} S e / Z n S e$ quantum dot", Superlattices and Microstructures, vol. 51, no. 1, pp. 184-193, 2012.

14. A. Tiutiunnyk, V. Tulupenko, M. E. Mora-Ramos, E. Kasapoglu, F. Ungan, H. Sari, I. Sökmen and C. A. Duque, "Electron-related optical responses in triangular quantum dots", Physica E, vol. 60, no. June, pp. 127-132, 2014.

15. W. Xie, "Impurity effects on optical property of a spherical quantum dot in the presence of an electric field", Physica B, vol. 405, no. 16, pp. 3436-3440, 2010.

16. E. Sadeghi, "Electric field and impurity effects on optical property of a three-dimensional quantum dot: A combinational potential scheme", Superlattices and Microstructures, vol. 50, no. 4, pp. 331-339, 2011.

17. L. He and W. Xie, "Effects of an electric field on the confined hydrogen impurity states in a spherical parabolic quantum dot", Superlattices and Microstructures, vol. 47, no. 2, pp. 266-273, 2010. 
18. H. Tąs and M. Şahin, "The inter-sublevel optical properties of a spherical quantum dotquantum well with and without a donor impurity", Journal of Applied Physics, vol. 112, no. 5, pp. 053717, 2012.

19. S. Yilmaz and M. Şahin, "Third-order nonlinear absorption spectra of an impurity in a spherical quantum dot with different confining potential", Physica Status Solidi B, vol. 247, no. 2, pp. 371-374, 2010.

20. İ. Karabulut, Ü. Atav, H. Şafak and M. Tomak, "Linear and nonlinear intersubband optical" absorptions in an asymmetric rectangular quantum well", European Physical Journal B, vol. 55, no. 3, pp. 283-288, 2007.

21. B. Çakir, Y. Yakar and A. Özmen, "Refractive index changes and absorption coefficients in a spherical quantum dot with parabolic potential", Journal of Luminescence, vol. 132, no. 10, pp. 2659-2664, 2012.

22. B. Çakir, Y. Yakar, A. Özmen, M. Özgür Sezer and M. Şahin, "Linear and nonlinear optical" absorption coefficients and binding energy of a spherical quantum dot", Superlattices and Microstructures, vol. 47, no. 4, pp. 556-566, 2010.

23. Y. Yakar, B. Çakir and A. Özmen, "Off-center hydrogenic impurity in spherical quantum dot" with parabolic potential", Superlattices and Microstructures, vol. 60, no. August 2013, pp. 389-397, 2013.

24. B. Çakir, Y. Yakar and A. Özmen, "Linear and nonlinear optical absorption coefficients of two-electron spherical quantum dot with parabolic potential", Physica B, vol. 458, no. 1 February 2015, pp. 138-143, 2015.

25. Z. Zeng, C. S. Garoufalis, A. F. Terzis and S. Baskoutas, "Linear and nonlinear optical properties of $Z n S / Z n O$ core shell quantum dots: Effect of shell thickness, impurity, and dielectric environment", Journal of Applied Physics, vol. 114, no. 2, 023510, 2013.

26. R. Khordad and H. Bahramiyan, "Impurity position effect on optical properties of various quantum dots", Physica E, vol. 66, no. February, pp. 107-115, 2015.

27. M. Kirak, S. Yilmaz, M. Şahin and M. Gençasian, "The electric field effects on the binding energies and the nonlinear optical properties of a donor impurity in a spherical quantum dot", Journal of App lied Physics, vol. 109, no. 9, pp. 094309, 2011.

28. G. Rezaei, M. R. K. Vahdani and B. Vaseghi, "Nonlinear optical properties of a hydrogenic impurity in an ellipsoidal finite potential quantum dot", Current Applied Physics, vol. 11, no. 2, pp. 176-181, 2011.

29. M. R. K. Vahdani and G. Rezaei, "Linear and nonlinear optical properties of a hydrogenic donor in lens-shaped quantum dots", Physics Letters A, vol. 373, no. 34, pp. 3079-3084, 2009.

30. M. J. Karimi and G. Rezaei, "Effects of external electric and magnetic fields on the linear and nonlinear intersubband optical properties of finite semi-parabolic quantum dots", Physica B, vol. 406, no. 23, pp. 4423-4428, 2011.

31. İ. Karabulut and S. Baskoutas, "Linear and nonlinear optical absorption coefficients and refractive index changes in spherical quantum dots: Effects of impurities, electric field, size, and optical intensity", Journal of Applied Physics, vol. 103, no. 7, pp. 073512, 2008.

32. S. Baskoutas, C. S. Garoufalis and A. F. Terzis, "Linear and nonlinear optical absorption coefficients in inverse parabolic quantum wells under static external electric field", European Physical Journal B, vol. 84, no. 2, pp. 241-247, 2011.

33. S. Baskoutas, E. Paspalakis and A. F. Terzis, "Electronic structure and nonlinear optical rectification in a quantum dot: effects of impurities and external electric field", Journal of Physics: Condensed Matters, vol. 19, no. 39, pp. 395024, 2007.

34. W. Xie, "Linear and nonlinear optical properties of a hydrogenic donor in spherical quantum dots", Physica B, vol. 403, no. 23-24, pp. 4319-4322, 2008.

35. W. Xie, "Nonlinear optical properties of a hydrogenic donor quantum dot", Physics Letters A, vol. 372, no. 33, pp. 5498-5500, 2008.

36. T. Chen, W. Xie and S. Liang, "Optical and electronic properties of a two-dimensional quantum dot with an impurity", Journal of Luminescence, vol. 139, no. July 2013, pp. 64-68, 2013.

37. K. M. Kumar, A. J. Peter and C. W. Lee, "Optical absorption and refractive index change of a confined exciton in a spherical quantum dot nanostructure" European Physical Journal B, vol. 84, no. 3, pp. 431-438, 2011.

38. R. Wei and W. Xie, "Optical absorption of a hydrogenic impurity in a disc-shaped quantum dot", Current Applied Physics, vol. 10, no. 3, pp. 757-760, 2010.

39. E. C. Niculescu, L. M. Burileanu, A. Radu and A. Lupaşcu, "Anisotropic optical absorption in quantum well wires induced by high-frequency laser fields", Journal of Luminescence, vol. 131, no. 6, pp. 1113-1120, 2011. 
40. E. C. Niculescu and L. M. Burileanu, "Nonlinear optical absorption in inverse $V$-shaped quantum wells modulated by high-frequency laser field", European Physical Journal B, vol. 74, no. 1, pp. 117-122, 2010.

41. G. Rezaei, B. Vaseghi, F. Taghizadeh, M. R. K. Vahdani and M. J. Karimi, "Intersubband optical absorption coefficient changes and refractive index changes in a two-dimensional quantum pseudodot system", Superlattices and Microstructures, vol. 48, no. 5, pp. 450-457, 2010.

42. G. H. Wang and K. -X. Guo, "Interband optical absorptions in a parabolic quantum dot", Physica E, vol. 28, no. 1, pp. 14-21, 2005.

43. A. Keshavarz and M. J. Karimi, "Linear and nonlinear intersubband optical absorption in symmetric double semi-parabolic quantum wells", Physics Letters A, vol. 374, no. 26, pp. 2675-2680, 2010.

44. M. G. Barseghyan, M. E. Mora-Ramos and C. A. Duque, "Hydrostatic pressure, impurity position and electric and magnetic field effects on the binding energy and photo-ionization cross section of a hydrogenic donor impurity in an InAs Pöschl-Teller quantum ring", European Physical Journal B, vol. 84, no. 2, pp. 265-271, 2011.

45. M. G. Barseghyan, A. A. Kirakosyan and C. A. Duque,"Donor-impurity related binding energy and photoionization cross-section in quantum dots: electric and magnetic fields and hydrostatic pressure effects", European Physical Journal B, vol. 72, no. December, pp. 521-529, 2009.

46. A. Hakimyfard, M. G. Barseghyan and A. A. Kirakosyan, "Simultaneous effects of pressure and magnetic field on intersubband optical transitions in Pöschl-Teller quantum well", Physica E, vol. 41, no. 8, pp. 1596-1599, 2009.

47. H. Yildirim and M. Tomak, "Optical absorption of a quantum well with an adjustable asymmetry", European Physical Journal B, vol. 50, no. 4, pp. 559-564, 2006.

48. İ. Karabulut and S. Baskoutas, "Second and third harmonic generation susceptibilities of spherical quantum dots: Effects of impurities, electric field and size", Journal of Computational and Theoretical Nanoscience, vol. 6, no. 1, pp. 153-156, 2009.

49. C. A. Duque, M. E. Mora-Ramos, E. Kasapoglu, F. Ungan, U. Yesilgul, S. Şakiroğlu, H. Sari and I. Sökmen, "Impurity-related linear and nonlinear optical response in quantum-well wires with triangular cross section", Journal of Luminiscence, vol. 143, no. November, pp. 304-313, 2013.

50. W. Xie,"Third-order nonlinear optical susceptibility of a donor in elliptical quantum dots", Superlattices and Microstructures, vol. 53, no. January, pp. 49-54, 2013.

51. W. Xie, "Optical anisotropy of a donor in ellipsoidal quantum dots", Physica B, vol. 407, no. 23, pp. 4588-4591, 2012.

52. T. Chen and W. Xie, "Nonlinear optical properties of a three-dimensional anisotropic quantum dot", Solid State Communications, vol. 152, no. 4, pp. 314-19, 2012.

53. Gh. Safarpour, M. A. Izadi, M. Novzari, E. Niknam and M. Moradi, "Anisotropy effect on the nonlinear optical properties of a three-dimensional quantum dot confined at the center of a cylindrical nano-wire", Physica E, vol. 59, no. May, pp. 124-132, 2014.

54. Gh. Safarpour, M. A. Izadi, M. Novzari and S. Yazdanpanahi, "Anisotropy effect on the linear and nonlinear optical properties of a laser dressed donor impurity in a GaAs/GaAlAs nanowire superlattice", Superlattices and Microstructures, vol. 75, no. November, pp. 936-937, 2014.

55. S. Rajashabala and K. Navaneethakrishnan, "Effects of dielectric screening and position dependent effective mass on donor binding energies and on diamagnetic susceptibility in a quantum well", Superlattices and Microstructures, vol. 43, no. 3, pp. 247-261, 2008.

56. S. Rajashabala and K. Navaneethakrishnan, "Effective masses for donor binding energies in quantum well systems", Modern Physics Letters B, vol. 20, no. 24, pp. 1529-1541, 2006.

57. S. Rajashabala and K. Navaneethakrishnan, "Effective masses for donor binding energies in non-magnetic and magnetic quantum well systems: effect of magnetic field", Brazilizn Journal of Physics, vol. 37, no. 3B, pp. 11341140, 2007.

58. A. J. Peter and K. Navaneethakrishnan, "Effects of position-dependent effective mass and dielectric function of a hydrogenic donor in a quantum dot", Physica E, vol. 40, no. 8, pp. 2747-2751, 2008.

59. R. Khordad, "Effects of position-dependent effective mass of a hydrogenic donor impurity in a ridge quantum wire", Physica E, vol. 42, no. 5, pp. 1503-1508, 2010.

60. R. Khordad, "Effect of position-dependent effective mass on linear and nonlinear optical properties of a cubic quantum dot", Physica B, vol. 406, no. 20, pp. 3911-3916, 2011. 
61. X. -H. Qi, X. -J. Kang and J. -J. Liu, "Effect of a spatially dependent effective mass on the hydrogenic impurity binding energy in a finite parabolic quantum well", Physical Review B, vol. 58, no. 16, pp. 10578-10582, 1998.

62. A. J. Peter, "The effect of position-dependent effective mass of hydrogenic impurities in parabolic GaAs/GaAlAs quantum dots in a strong magnetic field, "International Journal of Modern Physics B, vol. 23, no. 26, pp. 5109$5118,2009$.

63. Y. -X. Li, J. -J. Liu and X. -J. Kang, "The effect of a spatially dependent effective mass on hydrogenic impurity binding energy in a finite parabolic quantum well", Journal of Applied Physics, vol. 88, no. 5, pp. 2588-2592, 2000 .

64. Y. Naimi, J. Vahedi and M. R. Soltani, "Effect of position-dependent effective mass on optical properties of spherical nanostructures", Optical and Quantum Electronics, vol. 47, no. 8, pp. 2947-2956, 2015.

65. S. Pal, J. Ganguly, S. Saha and M. Ghosh, "Oscillator strength of impurity doped quantum dots: Influence of Gaussian white noise", Physica B, vol. 474, no. 1 October, pp. 41-46, 2015.

66. S. Sarkar, A. P. Ghosh, A. Mandal and M. Ghosh, "Modulating nonlinear optical properties of impurity doped Quantum dots via the interplay between anisotropy and Gaussian white noise", Superlattices and Microstructures, vol. 90, no. February, pp. 297-307, 2016.

67. S. Saha, S. Pal, J. Ganguly and M. Ghosh, "Influence of position-dependent effective mass on third-order nonlinear optical susceptibility of impurity doped Quantum dots in presence of Gaussian white noise", Physica B, vol. 484, no. 1 March, pp. 109-113, 2016.

68. A. P. Ghosh, A. Mandal, S. Sarkar and M. Ghosh, "Influence of position-dependent effective mass on the nonlinear optical properties of impurity doped Quantum dots in presence of Gaussian white noise", Optics Communications, vol. 367, no. 15 May, pp. 325-334, 2016.

69. V. Fock, "Bemerkung zur quantelung des harmonischen oszillators im magnetfeld", Zeitschrift für Physik, vol. 47, no. 5, pp. 446-448, 1928.

70. C. G. Darwin, "The diamagnetism of the free electron", Proceedings of Cambridge Philosophical Society, vol. 27, pp. 86,1930 . 